\section{Choroidal thickness in both eyes of patients with unilaterally active central serous chorioretinopathy}

YT Kim', SW Kang ${ }^{2}$ and KH Bai

\begin{abstract}
Objective To assess the change in the choroidal thickness of the unaffected eyes in patients with unilateral central serous chorioretinopathy (CSC). Methods Thirty eyes with unilateral idiopathic CSC and 30 age-matched normal eyes were included in this study. Choroidal thickness was evaluated from images obtained by enhanced depth image optical coherence tomography. The choroidal thicknesses of the affected eyes, unaffected eyes, and normal eyes were analyzed. Choroidal vascular dilation and hyperpermeability on indocyanine green angiography (ICGA) were analyzed and correlated with the changes in choroidal thickness.
\end{abstract}

Results The mean choroidal thicknesses of the affected eyes, unaffected fellow eyes, and normal individuals were $445.58 \pm 100.25$, $378.35 \pm 117.44$, and $266.80 \pm 55.45 \mu \mathrm{m}$, respectively. Compared with normal eyes, subfoveal choroidal thickness was increased significantly in the eyes with active CSC and in the unaffected fellow eyes $(P<0.001$ in both groups). The choroidal thickness was significantly greater in the eyes with active CSC than in the unaffected fellow eyes $(P=0.003)$. ICGA revealed choroidal vascular hyperpermeability in $28(93.3 \%)$ eyes with CSC and in $23(73.3 \%)$ unaffected fellow eyes. Choroidal vascular dilation was detected in $21(70.0 \%)$ eyes with CSC and in $18(60.0 \%)$ unaffected fellow eyes.

Conclusion Increased choroidal thickness in patients with unilateral CSC was noted not only in the affected eyes, but also in the unaffected fellow eyes. The results of this study suggest that CSC might be an essentially bilateral disorder.
Eye (2011) 25, 1635-1640; doi:10.1038/eye.2011.258; published online 21 October 2011

Keywords: central serous chorioretinopathy; fellow eye; choroidal thickness

\section{Introduction}

Central serous chorioretinopathy (CSC) is a relatively common disorder, which frequently involves bilateral involvement; bilaterality is reported in approximately $40 \%$ of cases. ${ }^{1}$

Although the pathogenesis of this condition has been extensively investigated, it remains a matter of controversy. In several hypotheses, choroidal hyperpermeability and dysfunction of RPE have been regarded as strong candidates for the origin of this disorder. ${ }^{2-4}$ The introduction of indocyanine green angiography (ICGA) supported the suggestion that choroidal vascular abnormality might be responsible for this condition. ${ }^{5,6}$ Hyperpermeability of the choroid shown on ICGA, a characteristic feature of CSC, may induce a functional loss of retinal pigment epithelial cells and abnormal egress of fluid under the retina.

Enhanced depth imaging optical coherence tomography (EDI-OCT), introduced by Spaide, provides more detailed tomographic images of the outer layers of the globe, particularly the choroid. ${ }^{7}$ Imamura et $a l^{8}$ reported that the choroidal thickness in eyes with CSC was significantly greater than that of normaleyes, as shown on EDI-OCT.

However, to the best of our knowledge, there has been no report thus far on the choroidal vascular thickness of the unaffected fellow eyes of patients suffering from unilateral active CSC. The principal objective of this study was to determine the changes in choroidal thickness of
${ }^{1}$ Department of Ophthalmology, Ewha Womans University, School of Medicine, Seoul, Republic of Korea

${ }^{2}$ Department of Ophthalmology, Samsung Medical Center, Sungkyunkwan University School of Medicine, Seoul, Republic of Korea

Correspondence: SW Kang, Department of Ophthalmology, Samsung Medical Center, Sungkyunkwan University School of Medicine, \#50 Irwon-dong, Gangnam-gu, Seoul 135-710, Republic of Korea

Tel: + 8223410 3562;

Fax: + 82234100074 .

E-mail: swkang@skku.edu

Received: 17 May 2011 Accepted in revised form: 16 August 2011 Published online: 21 October 2011 
the unaffected eyes as well as affected eyes of patients with unilateral CSC.

\section{Materials and methods}

Thirty patients with unilaterally active idiopathic CSC and 30 eyes of 30 normal individuals were included in the present study. The institutional review board approved the protocols of this study (SMC IRB No. 2010-07-208-001). Patients with a previous history of retinochoroidal disease other than idiopathic CSC were excluded, as were patients currently taking corticosteroids. Unilaterally active CSC was defined as the unilateral manifestation of active CSC at presentation, regardless of prior history of CSC in the affected or unaffected eyes. Thus, patients evidencing any presence of subretinal fluid or retinal pigment epithelium detachment in the fellow eyes at presentation were excluded from this study. In order to eliminate the potential influence of prior treatment on choroidal thickness, eyes that had undergone any vitreoretinal intervention, such as laser photocoagulation, photodynamic therapy, intravitreal injection, or vitreoretinal surgery, as well as eyes that had undergone cataract surgery within the past 6 months were also excluded. Patients with prior medical history of other vitreoretinal disorder, such as idiopathic polypoidal choroidal vasculopathy, retinitis, vasculopathy from retinal vein occlusion, or diabetic retinopathy, were not included.

Thirty eyes of 30 normal individuals without remarkable retinal and choroidal abnormality were recruited as a control group. Age and refractive errors were controlled to ameliorate unwanted bias from those compounding factors of choroidal thickness. ${ }^{9,10}$ Participants in this study included individuals aged between 30 and 65 years (Table 1). Subjects with high myopia, with refractive errors $>-6.0 \mathrm{D}$, were excluded. All of the participants in this study were Asian.

All eyes with CSC were examined using a slit lamp biomicroscope, fluorescein angiography, ICGA, and conventional spectral domain OCT (Spectralis, Heidelberg Engineering Inc., Heidelberg, Germany). For measurements of subfoveal choroidal thickness, $9 \mathrm{~mm}$ horizontal and vertical sections, which contained
100 duplicated scans, of the macula going through the foveola were obtained by the EDI-OCT maneuver previously described by Spaide. ${ }^{7}$ With these two sections, subfoveal choroidal thickness was manually measured using the contained Heidelberg eye explorer software (version 1.5.12.0, Heidelberg Engineering Inc.). Choroidal thickness was defined as the vertical distance from the hyperreflective line of Bruch's membrane to the innermost hyperreflective line of the chorio-scleral interface. Two averaged values of subfoveal choroidal thickness from the horizontal and vertical sections were analyzed. Choroidal vascular hyperpermeability and choroidal vascular dilation on ICGA were also assessed, and were correlated with the changes in choroidal thickness. Hyperpermeability was determined in the late-phase ICGA, and obtained between 8 to $15 \mathrm{~min}$ after dye injection as the presence of hyperfluorescent areas due to diffusion of the dye, detected as hyperfluorescent patches. Choroidal vascular dilation was defined as the focal or generalized dilation of the choroidal veins in the posterior pole on early-phase ICGA. An increase in choroidal thickness above the mean plus 2 standard deviation of that of the normal eyes was defined as statistically significant.

PASW software version 18.0 for Windows (SPSS, Inc., Chicago, IL, USA) was used for statistical analysis. Age and refractive errors were analyzed by two-tailed $t$-tests. Because of the hyperopic shift due to serous elevation in CSC, the refractive errors of the unaffected fellow eyes were compared. The Kolmogorov-Smirnov test was used to determine the normal distribution of choroidal thickness. The normal distribution of choroidal thickness was demonstrated in each group by the results of the Kolmogorov-Smirnov test $(P=1.00,0.54,0.89$, respectively, for affected eyes, fellow eye, and normal eyes). Thus, comparisons of the choroidal thickness of the eyes with CSC and unaffected fellow eyes with that of normal eyes were conducted by independent $t$-tests. Paired $t$-tests were also employed for the comparison of choroidal thickness of the eyes with CSC and that of unaffected fellow eyes. The $P$-values were adjusted by Bonferroni's correction in order to avoid the possible inflation of $P$-values owing to multiple comparisons.

Table 1 Demographic characteristics of normal individuals and patients with unilaterally active central serous chorioretinopathy

\begin{tabular}{lccc}
\hline & Normal individual & Patient with CSC & P-value \\
\hline No. of eyes & 30 & 30 & \\
Age, mean \pm SD (range), year & $48.2 \pm 14.0(27 \sim 69)$ & $48.2 \pm 8.1(31 \sim 68)$ & $1.00^{\mathrm{a}}$ \\
Sex (m:f) & $16: 14$ & $22: 8$ & $0.18^{\mathrm{b}}$ \\
Systemic hypertension, no. (\%) & $7(23.3)$ & $-0.5 \pm 1.5(-4.25$ to 1.50$)$ & $0.75^{\mathrm{b}}$ \\
Refractive error (range), D & $-1.3 \pm 2.2(-6.0$ to 2.0$)$ & $0.11^{\mathrm{a}}$ \\
\hline
\end{tabular}

Abbreviations: CSC, central serous chorioretinopathy; SE, spherical equivalent.

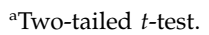

'Fisher's exact test. 
In order to avoid unwanted bias due to age and refractive errors, choroidal thickness was also evaluated by a general linear model with Bonferroni's correction. Data including sex and systemic hypertension were evaluated by Fisher's exact test and two-tailed $t$-tests were used to examine the relationship between choroidal thickness and clinical characteristics, including the presence of recurrence, symptom duration, and numbers of hyperfluorescent foci on ICGA. The correlation between symptom duration and choroidal thickness was evaluated by Pearson's correlation. Results were considered significant at $P$-values $<0.05$.

\section{Results}

The mean ages of normal individuals and patients with unilateral CSC were $48.2 \pm 14.0$ and $48.2 \pm 8.1$ years, and the mean refractive errors were $-1.3 \pm 2.2$ and $-0.5 \pm 1.5$, respectively (Table 1 ). No significant differences between the two groups were detected. The male-to-female ratio was 22:8 in the patients with CSC and 16:14 in normal individuals, and systemic hypertension was noted in $7(23.3 \%)$ patients with CSC and in $5(16.7 \%)$ normal individuals; sex ratio and systemic hypertension did not show statistical differences between the two groups $(P=0.18$ and 0.75 , respectively). Eleven patients had a prior history of CSC in the affected eye, and none of these patients underwent laser photocoagulation or photodynamic therapy. The mean duration of visual disturbance was $7.33 \pm 11.79$ weeks. One of the 30 CSC patients had a past medical history of CSC in the contralateral eye; however, no visual impairment or subretinal fluid was observed in the fellow eye at presentation.

The mean subfoveal choroidal thicknesses of the affected eyes, unaffected fellow eyes, and normal eyes were $445.58 \pm 100.25,378.35 \pm 117.44$, and $266.80 \pm 55.45 \mu \mathrm{m}$, respectively. The mean choroidal thicknesses of the affected eyes were significantly greater than those of the unaffected fellow eyes $(P<0.001)$ and normal eyes $(P<0.001)$. The mean choroidal thickness of the unaffected fellow eyes also significantly exceeded that of the normal eyes $(P=0.001)$. Comparison of choroidal thickness between these three groups by the general linear model also showed similar results; the choroidal thickness of each eye in the CSC patients was significantly greater, independently of refractive error and age, than that of normal individuals $(P<0.001$ in the affected eyes and $P<0.001$ in the unaffected fellow eyes). Additionally, the choroidal thickness of the CSC eyes was significantly larger than that of the unaffected fellow eyes, regardless of refractive errors and age $(P=0.003)$. However, significant correlation of the choroidal thickness with symptom duration was not found in either the affected eyes or the unaffected fellow eyes $(r=-0.237, P=0.216$ and $r=-0.332, P=0.079$, respectively).

ICGA revealed choroidal vascular hyperpermeability in $28(93.3 \%)$ eyes with CSC and $23(76.7 \%)$ unaffected fellow eyes, and choroidal vascular dilation was detected in $21(70.0 \%)$ eyes with CSC and $18(60.0 \%)$ unaffected fellow eyes (Table 2). A $>377.7 \mu$ m (mean plus 2 SD of normal eyes) increase in choroidal thickness was regarded as significant. Among 30 eyes with active CSC, 20 evidenced increase in both choroidal thickness and choroidal vascular hyperpermeability, and 15 eyes evidenced increase in both choroidal thickness and choroidal vascular dilation (Table 3). Meanwhile, among the affected eyes, choroidal vascular hyperpermeability was absent in two eyes and choroidal vascular dilation was not observed in seven eyes, despite evidence of increase in choroidal thickness (Table 3). Fifteen eyes showed increase in choroidal thickness in the unaffected fellow eyes. Among the eyes with increased choroidal thickness, choroidal vascular hyperpermeability was noted in 12 eyes and choroidal vascular dilation was observed in 9 eyes.

Prior history of recurrent CSC did not cause the change of choroidal thickness in either eyes with CSC or the fellow unaffected eyes. ( $P=0.631$ and 0.311 , respectively). Additionally, no significant correlation was noted to exist between choroidal thickness and duration of symptoms or numbers of hyperfluorescent foci on

Table 2 Subfoveal choroidal thickness and angiographic features of the affected eyes and unaffected fellow eyes

\begin{tabular}{|c|c|c|c|c|c|c|}
\hline & \multicolumn{2}{|c|}{ Increase of choroidal thickness $(>377.7 \mu \mathrm{m})^{\mathrm{a}}$} & \multicolumn{2}{|c|}{ Choroidal vascular hyperpermeability } & \multicolumn{2}{|c|}{ Choroidal vascular dilation } \\
\hline & Absent & Present & Absent & Present & Absent & Present \\
\hline Affected eyes, no. (\%) & $8(26.7)$ & $22(73.3)$ & $2(6.7)$ & $28(93.3)$ & $9(30.0)$ & $21(70.0)$ \\
\hline Fellow eyes, no. (\%) & $15(50.0)$ & $15(50.0)$ & $7(23.3)$ & $23(76.7)$ & $12(40.0)$ & $18(60.0)$ \\
\hline$P$-value ${ }^{\mathrm{b}}$ & \multicolumn{2}{|c|}{0.110} & \multicolumn{2}{|c|}{0.145} & \multicolumn{2}{|c|}{0.589} \\
\hline
\end{tabular}

Choroidal vascular hyperpermeability and vascular dilation were noted frequently not only in the affected eyes but also in the unaffected fellow eyes. Increase of choroidal thickness was also observed frequently in the unaffected fellow eyes as well as the affected eyes in subjects with unilaterally active CSC.

${ }^{a}$ Value of mean plus 2 standard deviation of choroidal thickness in normal individuals.

'Fisher's exact test. 
Table 3 The incidence of angiographic features according to subfoveal choroidal thickness in subjects with unilaterally active central serous chorioretinopathy

\begin{tabular}{|c|c|c|c|c|c|c|c|c|}
\hline \multirow[t]{3}{*}{ Choroidal thickness } & \multicolumn{4}{|c|}{ Affected eyes } & \multicolumn{4}{|c|}{ Unaffected fellow eyes } \\
\hline & \multicolumn{2}{|c|}{$\begin{array}{l}\text { Choroidal vascular } \\
\text { hyperpermeability }\end{array}$} & \multicolumn{2}{|c|}{$\begin{array}{c}\text { Choroidal vascular } \\
\text { dilation }\end{array}$} & \multicolumn{2}{|c|}{$\begin{array}{l}\text { Choroidal vascular } \\
\text { hyperpermeability }\end{array}$} & \multicolumn{2}{|c|}{$\begin{array}{c}\text { Choroidal vascular } \\
\text { dilation }\end{array}$} \\
\hline & Absent & Present & Absent & Present & Absent & Present & Absent & Present \\
\hline$\leq 377.7 \mu \mathrm{m}^{\mathrm{a}}$ & 0 & 8 & 2 & 6 & 4 & 11 & 6 & 9 \\
\hline$>377.7 \mu \mathrm{m}$ & 2 & 20 & 7 & 15 & 3 & 12 & 6 & 9 \\
\hline$P$-value ${ }^{\mathrm{b}}$ & \multicolumn{2}{|c|}{1.000} & \multicolumn{2}{|c|}{1.000} & \multicolumn{2}{|c|}{1.000} & \multicolumn{2}{|c|}{1.000} \\
\hline
\end{tabular}

Although increased choroidal thickness and angiographic abnormalities were noted more frequently in the affected eyes than in the unaffected fellow eyes simultaneously, change in choroidal thickness was not correlated with the presence of angiographic features.

${ }^{a}$ Value of mean plus 2 standard deviation of choroidal thickness in normal individuals.

'Fisher's exact test.

ICGA $(P=0.369,0.481$ in the eyes with CSC and 0.104, 0.173 in the unaffected fellow eyes).

\section{Discussion}

The results of this study revealed that choroidal thickness was significantly increased in the unaffected fellow eyes as well as affected eyes of CSC patients. The observed changes in choroidal thickness in the CSC eyes were consistent with the results of the previous study. ${ }^{8}$ The results of the current study also revealed a significant increase in choroidal thickness in the unaffected fellow eyes. Increased choroidal thickness in the CSC patients was independent of alleged compounding factors, age, and refractive errors.

There have been reports demonstrating the presence of hyperfluorescent plaques and choroidal venous dilation of ICGA in the unaffected fellow eyes of unilateral CSC patients. ${ }^{11}$ Comparing choroidal thickness with vascular features on ICGA, choroidal thickness corresponded well with the vascular changes shown on ICGA in most eyes with active CSC (Figure 1). That is, the mean subfoveal choroidal thickness was significantly greater in the eyes with active CSC than in the fellow eyes, and the incidence of hyperfluorescent plaque and vascular dilation on ICGA was also higher in the eyes with CSC than in the unaffected fellow eyes (28 vs 23 eyes for hyperfluorescent plaque and 21 vs 18 eyes for vascular dilation). However, in some cases, a discrepancy was noted between the increased choroidal thickness and the observed angiographic features. Although the reason for this discrepancy remains to be clarified, these findings may reflect certain phases in the clinical course of CSC. For instance, choroidal vascular dilation without extravasation may be a feature of the recovery phase, which precedes the absorption of extracellular exudates. Further longitudinal studies will be required to explain these conflicting features. Moreover, although we defined the angiographic features, the determination of angiographic features in each case was made rather subjectively. This may have caused some inaccuracy in the angiographic determinations, particularly in the marginal cases. The discrepancy in the tomography and angiography findings was more conspicuous in the unaffected fellow eyes than in the eyes with active CSC, which may be the result of the cut-off values of normal choroidal thickness. The authors established $377.7 \mu \mathrm{m}$ as the cut-off value for normal choroidal thickness, which may have been too large to determine accurately the increase in choroidal thickness in the unaffected fellow eyes. For a more precise discrimination of choroidal thickening, a larger number of normal subjects would also prove to be helpful.

The choroidal thickness of the normal individuals in this study was $266.80 \pm 55.45 \mu \mathrm{m}$. This value is similar to the results of a previous report by Spaide, $287 \pm 76 \mu \mathrm{m}$. In these studies, subfoveal choroidal thickness was evaluated by EDI-OCT. Recently, three-dimensional $1060 \mathrm{~nm}$ OCT was employed for the measurement of subfoveal choroids. ${ }^{12,13}$ In these studies, the normal values of subfoveal choroidal thickness were $354 \pm 111$ and $315 \pm 106 \mu \mathrm{m}$, respectively. The authors had suspected that ethnicity or different age distributions in these studies might result in different choroidal thickness values. However, considering the ethnic similarities between Koreans and Japanese, as well as the distribution of age in these studies, this discrepancy may have been the result of differences in measuring instruments and software.

Recently, Vance $e t$ al ${ }^{14}$ showed increase in choroidal thickness after administration of a selective inhibitor of cyclic guanosine monophosphate-specific phosphodiesterase-5, which acts as a vasodilator. Another study reported that choroidal thickness in patients with CSC decreased after photodynamic therapy, which targeted choroidal vasculature. ${ }^{15}$ These studies suggest that change in choroidal 

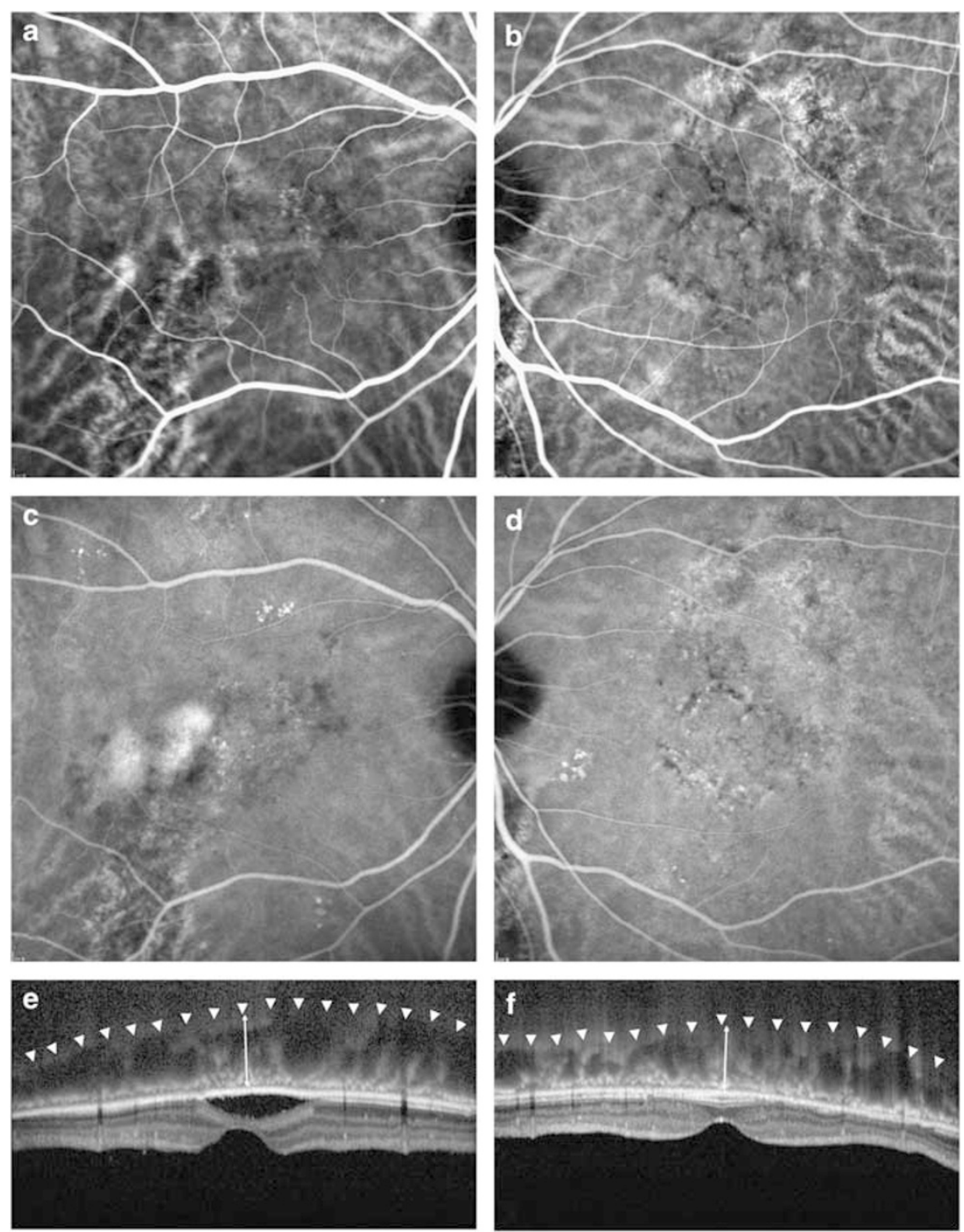

Figure 1 Representative ICGA and EDI-OCT of a patient with unilaterally active CSC. Choroidal vascular dilation and hyperpermeability on angiography and increased choroidal thickness on OCT were noted in the unaffected fellow eye (b, d, f), as well as in the eye with active CSC (a, c, e). Arrowheads indicate the inner sclera border and arrows demonstrate the estimated choroidal thickness from the outer border of the Bruch membrane to the inner scleral border.

thickness may be closely related to choroidal vasculature.

The increase in choroidal thickness noted in the fellow eyes of active CSC eyes suggests that this disorder may essentially be a bilateral or systemic disorder, which prevails over choroidal vasculature. The clinical characteristics of CSC, which include male predominance, relation to corticosteroid medication, or gestation, have been previously thought to indicate that this condition might not be confined to the retina or choroid. ${ }^{16-21}$

Choroidal vascular features on ICGA and choroidal thickening on OCT in the unaffected fellow eyes suggest that there may be some subclinical 'latent' phase of CSC that may precede the clinical manifestation of CSC. Also, this subclinical process may cause retinal pigment epithelial alterations and detachment or subretinal fluid accumulation by causing an increase in hydrostatic pressure. Broad retinal functional disturbance of both eyes in patients with unilateral involvement of CSC also suggests that CSC may be an essentially bilateral disorder. ${ }^{22}$

It remains unclear as to whether or not choroidal thickening on OCT may represent the activity of CSC. This, however, is beyond the scope of the current study. Further longitudinal follow-up is expected to reveal a relationship between tomographic changes and disease activity. If this is indeed the case, the assessment of choroidal thickness may prove to be a useful clinical indictor for the monitoring of CSC, as well as for 
diagnosing the disease. As several features indicate that CSC may be an ocular manifestation of an unknown systemic disorder, some effective pharmacological treatment may emerge. ${ }^{23}$ In this setting, the measurement of the choroid by EDI-OCT has several merits in the evaluation of CSC activity and responsiveness to treatment, including convenience, cost-effectiveness, non-invasiveness, and time saving.

The manual measurement of subfoveal choroidal thickness is one of the principal drawbacks of this study. Because current OCT equipment does not provide software for the automated measurement of choroidal thickness, all identifications of Bruch's membrane and the inner scleral border were conducted manually. However, considering the similarity of the normal values to those of the previous study, any bias deriving from the manual measurement is thought not to be significant. ${ }^{7,12,13}$

In conclusion, increased choroidal thickness in patients with unilateral CSC was noted not only in the affected eyes, but also in the unaffected fellow eyes. This thickness change appears to correlate with certain angiographic features, such as choroidal vascular hyperpermeability and dilation. The authors assume that this result supports the hypothesis that CSC is an essentially bilateral disorder.

\section{Summary}

What was known before

- Angiographic and electrophysiological changes on fellow eyes in patients with unilateral CSC has been alleged.

\section{What this study adds}

- Choroidal thickness increased not only in the affected eyes but also in the unaffected fellow eyes in patients with unilateral CSC.

\section{Conflict of interest}

The authors declare no conflict of interest.

\section{References}

1 Spaide RF, Campeas L, Haas A, Yannuzzi LA, Fisher YL, Guyer DR et al. Central serous chorioretinopathy in younger and older adults. Ophthalmology 1996; 103(12): 2070-2079; discussion 2079-2080.

2 Gass JD. Pathogenesis of disciform detachment of the neuro-epithelium. Am J Ophthalmol 1967; 63: 12.

3 Marmor MF. New hypotheses on the pathogenesis and treatment of serous retinal detachment. Graefes Arch Clin Exp Ophthalmol 1988; 226(6): 548-552.

4 Spitznas M. Pathogenesis of central serous retinopathy: a new working hypothesis. Graefes Arch Clin Exp Ophthalmol 1986; 224(4): 321-324.

5 Guyer DR, Yannuzzi LA, Slakter JS, Sorenson JA, Ho A, Orlock D. Digital indocyanine green videoangiography of central serous chorioretinopathy. Arch Ophthalmol 1994; 112(8): 1057-1062.

6 Spaide RF, Goldbaum M, Wong DW, Tang KC, Iida T. Serous detachment of the retina. Retina 2003; 23(6): 820-846; quiz 895-826.

7 Spaide RF, Koizumi H, Pozzoni MC. Enhanced depth imaging spectral-domain optical coherence tomography. Am J Ophthalmol 2008; 146(4): 496-500.

8 Imamura Y, Fujiwara T, Margolis R, Spaide RF. Enhanced depth imaging optical coherence tomography of the choroid in central serous chorioretinopathy. Retina 2009; 29(10): 1469-1473.

9 Fujiwara T, Imamura Y, Margolis R, Slakter JS, Spaide RF. Enhanced depth imaging optical coherence tomography of the choroid in highly myopic eyes. Am J Ophthalmol 2009; 148(3): 445-450.

10 Margolis R, Spaide RF. A pilot study of enhanced depth imaging optical coherence tomography of the choroid in normal eyes. Am J Ophthalmol 2009; 147(5): 811-815.

11 Iida T, Kishi S, Hagimura N, Shimizu K. Persistent and bilateral choroidal vascular abnormalities in central serous chorioretinopathy. Retina 1999; 19(6): 508-512.

12 Ikuno Y, Kawaguchi K, Nouchi T, Yasuno Y. Choroidal thickness in healthy Japanese subjects. Invest Ophthalmol Vis Sci 2010; 51(4): 2173-2176.

13 Esmaeelpour M, Povazay B, Hermann B, Hofer B, Kajic V, Kapoor K et al. Three-dimensional $1060 \mathrm{~nm}$ OCT: choroidal thickness maps in normals and improved posterior segment visualization in cataract patients. Invest Ophthalmol Vis Sci 2010; 51(10): 5260-5266.

14 Vance SK, Imamura Y, Freund KB. The effects of sildenafil citrate on choroidal thickness as determined by enhanced depth imaging optical coherence tomography. Retina 2011; 31(2): 332-335.

15 Maruko I, Iida T, Sugano Y, Ojima A, Ogasawara M, Spaide RF. Subfoveal choroidal thickness after treatment of central serous chorioretinopathy. Ophthalmology 2010; 117(9): 1792-1799.

16 Garg SP, Dada T, Talwar D, Biswas NR. Endogenous cortisol profile in patients with central serous chorioretinopathy. Br J Ophthalmol 1997; 81(11): 962-964.

17 Gass JD. Central serous chorioretinopathy and white subretinal exudation during pregnancy. Arch Ophthalmol 1991; 109(5): 677-681.

18 Gass JD, Little H. Bilateral bullous exudative retinal detachment complicating idiopathic central serous chorioretinopathy during systemic corticosteroid therapy. Ophthalmology 1995; 102(5): 737-747.

19 Haimovici R, Koh S, Gagnon DR, Lehrfeld T, Wellik S. Risk factors for central serous chorioretinopathy: a case-control study. Ophthalmology 2004; 111(2): 244-249.

20 Tittl MK, Spaide RF, Wong D, Pilotto E, Yannuzzi LA, Fisher YL et al. Systemic findings associated with central serous chorioretinopathy. Am J Ophthalmol 1999; 128(1): 63-68.

21 Menchini U, Virgili G, Lanzetta P, Ferrari E. Indocyanine green angiography in central serous chorioretinopathy. ICG angiography in CSC. Int Ophthalmol 1997; 21(2): 57-69.

22 Marmor MF, Tan F. Central serous chorioretinopathy: bilateral multifocal electroretinographic abnormalities. Arch Ophthalmol 1999; 117(2): 184-188.

23 Tatham A, Macfarlane A. The use of propranolol to treat central serous chorioretinopathy: an evaluation by serial OCT. J Ocul Pharmacol Ther 2006; 22(2): 145-149. 